\title{
Arborescences
}

Revue d'études françaises

\section{De la norme grammaticale du français parlé au Québec}

\section{Davy Bigot}

Numéro 1, mars 2011

Identités linguistiques, langues identitaires : à la croisée du prescriptivisme et du patriotisme

URI : https://id.erudit.org/iderudit/1001939ar

DOI : https://doi.org/10.7202/1001939ar

Aller au sommaire du numéro

\section{Éditeur(s)}

Département d'études françaises, Université de Toronto

ISSN

1925-5357 (numérique)

Découvrir la revue

Citer cet article

Bigot, D. (2011). De la norme grammaticale du français parlé au Québec. Arborescences, (1), 0-0. https://doi.org/10.7202/1001939ar

\section{Résumé de l'article}

Dans le cadre de cet article, je me propose d'examiner l'usage de quatorze variables linguistiques dans le discours de personnes faisant partie des élites québécoises. Tout d'abord, je reviendrai sur l'ensemble du débat de la norme linguistique au Québec, et plus spécifiquement sur le problème de la norme grammaticale orale. Ensuite, j'exposerai les principaux points méthodologiques de mon étude. Enfin, je démontrerai, qu'en général, le parler soigné des élites sociale et culturelle québécoises placées en situation de communication formelle correspond au modèle du français de référence décrit entre autres dans Le bon usage de Grevisse-Goosse. d'utilisation que vous pouvez consulter en ligne.

https://apropos.erudit.org/fr/usagers/politique-dutilisation/ 


\title{
DE LA NORME GRAMMATICALE \\ DU FRANÇAIS PARLÉ AU QUÉBEC
}

\author{
Davy Bigot \\ Université Concordia
}

\section{Introduction}

Près de trente ans après la création de l'Office de la langue française, le Conseil de la langue française (CLF, 1990) soulignait toujours l'importance de décrire le français standard du Québec. Depuis, Cox (1998) et Reinke (2005) ont démontré qu’il existe effectivement un modèle standard québécois de prononciation en usage dans les médias. Sur le plan lexical, plusieurs dictionnaires ont été publiés (entre autres, Boulanger et Rey 1992 ; Meney 1999 ; de Villers 2003), mais ils ont tous fait l'objet de diverses critiques (idéologiques et/ou méthodologiques). Bien que le débat du dictionnaire soit loin d'être clos (le projet du Franqus ${ }^{1}$ a été condamné plusieurs fois par Lionel Meney (2003, 2005a, 2005b, 2010), on peut toutefois considérer que le lexique du français québécois est bien documenté.

L'intérêt envers le problème de la norme grammaticale orale a, quant à lui, été très limité. Certains spécialistes pensent qu'il existe une norme québécoise différente de celle de la France ; d'autres affirment que cette norme est au contraire identique au modèle international. Enfin, selon Barbaud (1998a, 1998b), l'élite québécoise, détentrice de la norme linguistique, utilise à l'oral des structures syntaxiques beaucoup trop divergentes pour pouvoir élaborer une norme du français québécois.

Dans cet article, je me propose d'examiner quatorze variables grammaticales au sein d'un corpus de cent dix locuteurs. Dans un premier temps, j'exposerai les différentes propositions concernant ce qu'est ou devrait être la norme du français québécois, et plus particulièrement sur son aspect grammatical oral. Je présenterai ensuite les principaux points méthodologiques de mon étude. Enfin, je démontrerai qu'en général, la norme grammaticale du parler des élites sociale et culturelle québécoises placées en situation de communication formelle n'est pas très éloignée de celle décrite dans Le bon usage de Grevisse-Goosse.

\footnotetext{
${ }^{1}$ Le groupe de recherche Français Standard en Usage au Québec de l'Université de Sherbrooke travaille depuis 2001 à l'élaboration d'un dictionnaire du français québécois contemporain d'usage public (http://franqus.ca/projet/).
} 


\section{Le débat sur la norme du français québécois}

Depuis le début des années 60, plusieurs définitions ont été proposées. Deux positions s'affrontent: la première considère que la norme du français québécois doit s’aligner sur le modèle international, la seconde considère qu'il existe une norme typiquement québécoise.

\section{- Une norme basée sur le modèle international}

En 1965, l'Office de la langue française (désormais OLF) publie un document consacré à la norme du français écrit et parlé au Québec. Une première définition de la norme est donnée: « [Celle-ci doit] coïncider à peu près entièrement avec celle qui prévaut à Paris, Genève, Bruxelles, Dakar et dans toutes les grandes villes d'expression française » (Ministère des Affaires culturelles du Québec 1965 : 6). En faisant référence à une variété commune à quatre grandes capitales de la francophonie, l'OLF prend position en faveur d'un modèle normatif international. Dans ce document, on peut également lire que : «La norme ainsi conçue doit s'étendre à tous les aspects de la langue : morphologie, syntaxe, phonétique, lexique ; mais pour ce qui est des deux premiers, qui sont d'ordre structural, la variation doit être inexistante» (Ministère des Affaires culturelles du Québec 1965 : 6). La position de l'OLF est très claire : la grammaire normative du français québécois (écrite/orale) ne peut s'éloigner du français international.

Cette vision est toujours partagée par plusieurs spécialistes. Corbeil (1993 : 28) déclare que : «La morphologie fait partie du noyau dur de la langue. Aucune variation n'est admise ». Il ajoute « [qu'il] n'existe aucune différence notable entre la syntaxe du français québécois et celle du français français » (Corbeil 1993 : 29). Nemni (1998 : 160) affirme aussi que : « Nous savons tous que les plus grands écarts entre le français québécois et le français standard se situent dans les registres familiers et populaires». Elle ajoute un peu plus loin que «les Québécois francophones utilisent le français international pour le registre formel » (Nemni 1998 : 167). Cette position sera reprise à l'identique

(ou presque) dans le rapport Larose en 2001 (Commission des États généraux sur la situation et l'avenir de la langue française au Québec 2001 : 84) sans qu'aucune donnée ne vienne l'appuyer.

Dans son dernier ouvrage, Corbeil (2007 : 316) fait une remarque très intéressante : "Pour être complète, la description de la norme du français standard québécois devrait toucher les trois composantes de ce français, la prononciation, la grammaire (morphologie et syntaxe) et le lexique ». Il souligne néanmoins que : «l'écart entre la morphologie et la syntaxe de la langue parlée au Québec et la norme de la langue écrite est variable. L'écart est pour ainsi dire nul en langue parlée soignée » 
(Corbeil 2007 : 320). Si cela est évident pour Corbeil, il me semble que des données sont nécessaires pour illustrer son point de vue. L'auteur n'en fournit cependant aucune.

Enfin, le président du Conseil supérieur de la langue française (désormais CSLF) déclarait que : «le seul français qui peut être enseigné est un français national, de niveau international, qui peut être défini différemment, selon qu'il s'agit de l'oral ou de l'écrit. Le français d'ici, de registre « officiel», correspond à ce français international»(CSLF 2007 : 2). Malgré l'apparente clarté de cette affirmation, de nombreuses zones d'ombre subsistent. Celle-ci soulève effectivement plusieurs questions. Qu'entend-on par français de niveau international? Quel est ce français d'ici de registre officiel? Que doit-on comprendre par registre « officiel»? Quels sont les locuteurs susceptibles de parler ce français officiel?

\section{- Une norme « à la québécoise »}

Depuis la querelle du joual, les enseignants ont été profondément divisés sur le choix de la norme du français à enseigner dans les écoles (Comité interministériel sur la situation de la langue française 1996 : 183). En 1977, l’Association québécoise des professeurs de français (désormais AQPF) débat autour des questions suivantes : « quel est le français standard d'ici ? » et « quel français doit-on enseigner au Québec?». Les membres concluent que : «Le français standard d'ici est la variété de français socialement valorisée que la majorité des Québécois francophones tendent à utiliser dans les situations de communication formelle » (AQPF $1977: 11)^{2}$. La définition de l'AQPF paraît très simple, mais elle pose selon moi une question majeure : quel est ce français socialement valorisé par les Québécois ${ }^{3}$ ?

Selon Forget (1979) et Cajolet-Laganière et Martel (1995) (repris par Lepicq et Bourhis 1995), il existe bel et bien une norme québécoise. Cajolet-Laganière et Martel (1995 : 158) affirment : « un modèle linguistique français, mais français québécois, fait consensus au Québec. [...] Or ce modèle québécois n'est décrit nulle part $»^{4}$. Cette position s'oppose radicalement à celle préconisant une norme du français québécois basée sur un français standard international.

\footnotetext{
${ }^{2}$ Cette définition est d'ailleurs reprise treize ans plus tard par le CLF (CLF 1990 : 31).

${ }^{3}$ Le français des annonceurs de nouvelles de Radio-Canada serait le plus valorisé selon Maurais (1999) et Bouchard et Maurais (2001), mais il n'est pas sans poser des problèmes (Bigot 2008 : 133).

${ }^{4}$ Je remercie Hélène Cajolet-Laganière de m'avoir précisé que par « modèle linguistique », elle n’incluait pas la grammaire orale. Je tiens toutefois à souligner que l'expression même de "modèle linguistique » porte à confusion, car elle laisse entendre que les auteurs incluent le lexique, la prononciation et aussi la grammaire.
} 


\section{- La concurrence de deux variétés de français au Québec}

Selon Philippe Barbaud, le problème se pose de façon plus complexe. Il indique que «le parler d'ici s'est engagé sur une trajectoire « séparatiste » par rapport au français qui se parle ailleurs dans le monde » (1987a : 159). Cet éloignement de la norme du français québécois oral par rapport à celle des autres variétés de la langue française, s'explique à partir d'une constatation que Barbaud (1987b : 66) formule ainsi :

Le français du Québec tel qu'il est parlé par les masses parlantes, qu'elles soient populaires ou simili-bourgeoises, est d'une remarquable homogénéité phonétique, syntaxique et stylistique. Nos différences régionales s'estompent et se fondent toutes dans un modèle commun qui nous rassemble parce qu'il nous ressemble [...]

Dans deux articles publiés plus tard, Barbaud (1998a, 1998b) reprend sa théorie et propose une analyse basée sur les usages grammaticaux de l'élite québécoise. Son objectif est très clair :

Je me propose de montrer que le français du Québec (FQ) se révèle suffisamment divergent dans l'usage qui est propre aux élites d'ici, sur le plan syntaxique, pour que toute tentative d'élaborer une norme authentiquement québécoise de français québécois standard (en abrégé FQS) conduise inévitablement à une impasse généralisée sur le plan communicationnel. (Barbaud 1998 : 107)

Pour cela, il recueille des données sur les usages de l'élite québécoise (Barbaud 1998a: 110, note infrapaginale 2). Il conclut de ses observations que :

Que l'on préconise une syntaxe du FQS alignée sur celle du FSI [français standard international] ou, au contraire, alignée sur l'usage qu'en font les élites québécoises, on s'engage dans une impasse : celle de trahir la réalité langagière d'ici ou de l'ignorer. Réalité de la divergence québécoise ou réalité de la dominance hexagonale. (Barbaud 1998a : 14)

Je ferai à l'égard de son analyse les critiques suivantes. Celle-ci se base sur des faits de langue, certes constatés, mais non regroupés dans un corpus sociolinguistique dûment constitué ${ }^{5}$. À aucun moment, l'auteur ne nous renseigne sur la fréquence des diverses tournures syntaxiques qu'il a notées. Il n’indique pas non plus si le locuteur s'est auto-corrigé ou non. On peut alors se poser la question de savoir si ces tournures sont réellement légitimées par les élites québécoises ou si elles ne sont pas simplement des tournures dispersées dans des discours de locuteurs qui maîtriseraient très bien la grammaire standard. Barbaud (1987a: 157) précise d'ailleurs que : "Pour déterminer si, à l'oral, une forme quelconque est standard ou non, il convient d'établir qu'elle réponde au critère de l'emploi fréquent, généralisé et acceptable [...] ». Il me semble que les données de Barbaud (1998a, 1998b) ne nous permettent absolument pas d'atteindre cet objectif.

\footnotetext{
${ }_{5}^{5}$ J'entends par corpus sociolinguistique, une base de données linguistiques représentative de l'usage réel et typique de la population observée permettant une analyse statistique.
} 
Son analyse n'en est pas pour autant inintéressante. L'auteur était jusqu’à maintenant le seul à avoir réellement soulevé le problème de la concurrence des formes vernaculaires et standard dans le français parlé par les élites québécoises. En cela, son étude reste précieuse et mérite toute notre attention. De plus, elle permet de comprendre que les structures grammaticales typiques du français québécois vernaculaire sont le fruit de règles précises et cohérentes, et non d'une détérioration de la langue française ${ }^{6}$.

\section{La méthodologie}

Mon objectif est de rendre compte des usages grammaticaux réels de l'élite sociale et culturelle québécoise en situation de communication formelle. Pour cela, j'ai rassemblé plusieurs entrevues journalistiques diffusées dans l'émission Le Point qui, jusqu'en juin 2006, faisait suite au Téléjournal de vingt-deux heures à Radio-Canada (ce corpus d'entrevues s'appelle naturellement le corpus «Le Point »). Mon choix s'explique ainsi : 1) le lieu de l'entrevue est le plateau de l'émission Le Point (cadre formel de communication assurant un degré d'autosurveillance et d'autocorrection maximal chez l'interviewé), 2) l'interviewé fait partie des élites québécoises (Barbaud, 1998a: 110, note infrapaginale 2), et 3) les sujets traités dans ces entrevues sont abordés de façon très formelle (ex : les entrevues abordant le domaine du sport traitent de problèmes d'arbitrage ou encore de dopage).

J'ai choisi de ne considérer qu'un seul type d'entrevue journalistique, dans le but de réduire au maximum l'apparition de variables parasites : l'intervieweur et l'interviewé sont présents sur le plateau de l'émission. J'ai exclu toute entrevue multimédia où l'interviewé fait face à une caméra. Je n'ai pas pris en compte les entrevues durant lesquelles plus d'une seule personne est interviewée (cela me permettait d'éviter d'éventuelles interactions pouvant favoriser la présence de formes grammaticales vernaculaires). Par ailleurs, je n'ai opéré aucune sélection des invités, outre le fait que j'ai exclu ceux qui n'étaient pas Québécois francophones d'origine. Cette information m'a été fournie de deux façons. Lorsque la personne était une personnalité connue, j’ai déterminé son origine à partir d'une recherche sur Internet. Lorsque ce n'était pas le cas, j'ai fait appel à des Québécois d'origine afin de m'assurer que la personne pouvait être retenue dans mon corpus. Bien entendu, il me faut ici admettre que mes informateurs (deux hommes et une femme âgés entre 25 et 30 ans) ont pu commettre des erreurs. Toutefois, pour les quelques cas problématiques que j'ai rencontrés, leurs

\footnotetext{
${ }^{6}$ Voir également à ce sujet Léard (1995).
} 
réponses étaient identiques et sans hésitation. Au final, le corpus est constitué de cent dix entrevues d'environ dix minutes pour un total de seize heures d'enregistrement.

J'ai tenu compte des facteurs sociaux sexe, âge et catégorie d'emploi afin de vérifier si un ou plusieurs groupes de locuteurs se distinguaient des autres dans l'utilisation de variantes vernaculaires. Le tableau 1 présente la répartition des interviewés par variable sociale.

Tableau 1 : Répartition des interviewés par variable sociale

\begin{tabular}{|l|c|c||}
\hline Variable sociale & Pourcentage (\%) & Nombre d'interviewés \\
\hline \hline Sexe & & \\
\hline Hommes & 82 & 90 \\
\hline Femmes & 18 & 20 \\
\hline Áge & & \\
\hline 61 ans et plus & 27,2 & 30 \\
\hline 31 ans à 60 ans & 71 & 78 \\
\hline 30 ans et moins & 1,8 & 2 \\
\hline Catégorie d'emploi & & 25 \\
\hline Politiciens & 23 & 22 \\
\hline Responsables & 20 & 20 \\
\hline Universitaires & 18 & 14 \\
\hline Artistes & 13 & 11 \\
\hline Juges/Avocats & 10 & 10 \\
\hline Médecins & 9,5 & 3 \\
\hline Hauts gradés (Armée/Police) & 2,5 & \\
\hline Élite sportive & & \\
\hline Éminences religieuses & & \\
\hline \hline
\end{tabular}

J'ai retenu les quatorze variables présentées ci-dessous ${ }^{8}$. La première variante est de type vernaculaire, la seconde est de type standard. Chaque variable est suivie de sa référence dans le Bon

${ }^{7}$ Cette catégorie renvoie aux cadres, aux cadres supérieurs et aux chefs d'entreprises.

8 Ces variables ne sont pas toutes stigmatisées au même titre. Par exemple, la prononciation de je vais en j'vas est nettement plus stigmatisée que la neutralisation du présentatif ce sont des... en c'est des... 
usage de Grevisse-Goosse (2008). Viennent ensuite les références aux études antérieures consultées pour notre étude.

1) La prononciation de tous/tout en / tut/ ${ }^{9}$, p. ex. : Ils sont / tut/ vs / tus/ venus ; (Grevisse-Goosse 2008: 815), (Lemieux-Nieger, Leblanc et Paquin 1981; Lemieux 1982; Lemieux et Sankoff 1983; Lemieux, St-Amour et Sankoff 1985 ; Cyr 1991 ; Léard et Beauchemin 1991 ; Junker 1994, 1995 ; De Cat 2000, Bélanger 2003 ; Daveluy 2005).

2) La prononciation des démonstratifs ce, cet, cette en [sta], p. ex. : [sta] vs [sEt] voiture là ; (Grevisse-Goosse 2008 : 794), (Daveluy 1987, 2005 ; Léard 1995).

3) La prononciation du participe passé de faire en [fet], p. ex. : Il a [fet] vs $[f \varepsilon]$ son travail; (Grevisse-Goosse 2008 : 1168 et 1175), (Léard 1995 ; Daveluy 2005).

4) La prononciation de je vais en j'vas ou m'as, p. ex. : J'vas/m'as travailler vs je vais/j'vais travailler; (Grevisse-Goosse 2008: 1042 et 1058), (Léard 1995; Mougeon 1994). Il est à noter que le verbe «aller» n'a été retenu que lorsque sa fonction était celle de semi-auxiliaire.

5) L'insertion du l' non étymologique, p. ex. : Ça l' vs o en prend beaucoup...; (Grevisse-Gosse 2008 : 894), (Morin 1982).

6) La double négation, p. ex. : Il y a pas vs o personne/rien; (Grevisse-Goosse 2008 : 1283), (Léard 1995).

7) La locution conjonctive quand suivi de que, p. ex. : Quand que vs o je rentre chez. moi... ; (Grevisse-Goosse 2008 : 1484), (Léard 1995).

8) Les relatives indirectes en que, p. ex. : C'est la personne que vs dont je te parlais; (Grevisse-Goosse 2008 : 926), (Léard 1995 ; Barbaud 1998a, 1998b ; Remysen 2003 ; Ostiguy, Champagne, Gervais et Lebrun 2005 ; Corbeil 2007)

9) L'emploi du futur périphrastique dans les phrases affirmatives, p. ex. : Je vais le voir vs je le verrai demain; (Grevisse-Goosse 2008: 1042), (Deshaies et Laforge 1981 ; Emirkanian et Sankoff 1985 ; Zimmer 1994 ; Poplack et Turpin 1999 ; Sankoff et Wagner 2006).

10) L'emploi de l'auxiliaire avoir avec les verbes de mouvement, p. ex. : Elle a passé vs est passée par Montréal; (Grevisse-Goosse 2008: 1032), (Sankoff et Thibault 1977 ; Léard 1995)

11) L'emploi du conditionnel après si, p. ex. : Si j'aurais vs j'avais fait mon travail...; (Grevisse-Goosse 2008 : 1092), (Lavoie 1989 ; Léard 1995).

\footnotetext{
${ }^{9}$ Voir Bigot (2008 : 211-226) pour plus de détails sur le décompte de tout/tous.
} 
12) Le présentatif en c'est des, p. ex. : C'est des vs ce sont des personnes très sympathiques;

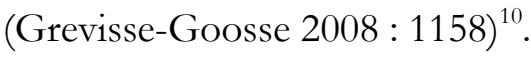

13) Les complétives et interrogatives indirectes, p. ex. : Je sais pas que c'est que/qu'est-ce que vs ce que je vais faire. et Je me demande que c'est que/qu'est-ce que vs ce que je vais faire ; (Grevisse-Goosse 2008 : 1527), (Kemp 1979 ; Léard 1995).

14) Les interrogatives (totales et partielles) $)^{11}$, p. ex. : Tu y vas-tu?/Tu y vas ? vs Y-vastu?/Est-ce que th y vas? et Que c'est que tu en penses?/Que c'est que c'est que t'en penses?/Qu'est-ce t'en penses? vs Qu'en penses-tu?/Qu'est-ce que tu en penses?; (Grevisse-Goosse 2008 : 486), (Barbarie 1982 ; Léard 1995).

Donner simplement le nombre d'occurrences des formes vernaculaires n'apporterait pas grande information sur leur utilisation. Maleville (1982: 70) remarquait qu' « établir des normes, c'est comparer». Il était donc nécessaire de comparer le nombre d'occurrences de chaque forme vernaculaire avec celui des formes propres à un français plus normatif, dans le cas présent, les formes équivalentes régies par les règles décrites dans la quatorzième édition du Bon usage de Grevisse-Goosse.

Cet ouvrage représente le modèle grammatical de référence pour une grande partie de la francophonie. Certes, c'est une grammaire de l'écrit, mais elle représente aussi, selon moi, une forme acrolectale, voire hyperlectale (au sens de Chaudenson 1997 : 19) de la grammaire orale. De plus, il n'existe encore à ce jour aucune description normative de la grammaire du français oral. Enfin, les études de Maurais (2005) ou encore celles d'Ostiguy, Champagne, Gervais et Lebrun (2005) utilisent aussi la grammaire de Grevisse-Goosse comme modèle de référence. Ceci n'est pas un hasard puisqu'elle représente un modèle de code normé connu et commun, de registre formel, à partir duquel il est possible de dégager des points de convergence et de divergence entre les variétés de français écrit et parlé.

\section{Présentation des résultats généraux}

J'ai recueilli un total de 2652 formes grammaticales (vernaculaires et standard) réparties sur l'ensemble des quatorze variables. Les pourcentages des variantes vernaculaires utilisées dans le corpus sont présentés dans le tableau 2.

\footnotetext{
${ }^{10}$ Dans le cas du présentatif en c'est des..., aucune étude sur le phénomène en français québécois n'a été trouvée.

11 Voir Bigot (2008 : 227-243) pour une analyse interne des interrogatives directes prises en compte dans mon étude.
} 
Tablean 2 : Pourcentages des variantes vernaculaires produites par variable ${ }^{12}$

\begin{tabular}{|l|c|c||}
\hline Variable linguistique & $\mathbf{\%}$ & $\mathbf{n} / \mathbf{N}$ \\
\hline \hline Emploi du futur périphrastique à l'affirmatif & 78,9 & $564 / 715$ \\
\hline Le présentatif en c'est des... & 60,4 & $87 / 144$ \\
\hline Les interrogatives directes & 20,4 & $49 / 240$ \\
\hline Réalisation de je vais en j'vas/ m'as + verbe à l'infinitif & 20,3 & $17 / 84$ \\
\hline Prononciation du participe passé fait en [fet] & 10,1 & $24 / 237$ \\
\hline Emploi de que dans les relatives indirectes & 9,5 & $5 / 52$ \\
\hline Emploi de la double négation avec rien / personne & 8,1 & $5 / 62$ \\
\hline Insertion du / non étymologique devant ça & 8 & $11 / 138$ \\
\hline Le conditionnel employé après si & 5,7 & $4 / 70$ \\
\hline Emploi de l'auxiliaire avoir devant les verbes de mouvement & 5,5 & $7 / 128$ \\
\hline Les structures complétives et interrogatives indirectes & 4,6 & $20 / 435$ \\
\hline Emploi de la conjonction quand... suivi de que & 3,5 & $9 / 258$ \\
\hline Prononciation de tout/ tous en [tut] & 2,9 & $13 / 450$ \\
\hline Prononciation des démonstratifs ce et cette / cet en [st(ə)] & 1,5 & $8 / 528$ \\
\hline
\end{tabular}

La colonne 1 présente la forme vernaculaire de la variable. La colonne 2 présente le pourcentage d'occurrences de cette dernière. La colonne 3 présente le nombre (n) d'occurrences de la variante vernaculaire sur le nombre total de formes $(\mathrm{N})$.

Le tableau 2 permet de dégager les observations suivantes. Tout d'abord, les variables « Emploi du futur périphrastique à l'affirmatif » et «Le présentatif en c'est des » obtiennent les scores les plus importants $(78,9 \%$ pour la première et $60,4 \%$ pour la seconde) et se détachent nettement des douze autres variables. Il faut également distinguer les deux variables «Les interrogatives directes» et «Réalisation de je vais en j'vas/m'as + verbe à l’infinitif» dont les chiffres sont de 20,4 \% et 20,3\%. Je précise dès maintenant deux points importants concernant ces deux dernières variables. Les 20,4\% d'interrogatives directes de type vernaculaire ont été produits majoritairement par la catégorie des artistes (soit $13 \%$ des locuteurs) qui emploient une de ces structures dans $31 \%$ des cas (contre une moyenne de 5,5\% pour les huit autres catégories). Ces 20,4\% de formes vernaculaires des interrogatives directes sont donc davantage dus au résultat d'un groupe en particulier qu'à

\footnotetext{
12 Les résultats concernant la variation observée pour chacune des variables en fonction des facteurs linguistiques et extralinguistiques sont détaillés dans Bigot (2008).
} 
l'ensemble des locuteurs de notre corpus. Pour la réalisation de je vais en j'vas/m'as, je n'ai recueilli aucune forme en m'as. De plus, les 20,3\% de j'vas n'ont été produits que par $8 \%$ des locuteurs de notre corpus. Ce résultat est donc aussi davantage le fruit d'un groupe de locuteurs hétérogène (ces derniers appartenant à la catégorie des politiciens, des universitaires ou des élites sportives et étant âgés de plus de 31 ans) que de l'ensemble des interviewés. En conséquence, je pense qu'à l'exception des variables «Emploi du futur périphrastique au positif» et «Le présentatif en c'est des », les formes standard sont très nettement privilégiées par les locuteurs du corpus.

Concernant l'emploi du futur périphrastique dans les phrases affirmatives, je pense que bien que cette forme de futur ne soit pas officiellement prescrite en français standard, elle reste la norme officieuse au Québec, car aucun des facteurs externes âge, sexe et catégorie d'emploi ne s'est révélé significatif (Bigot 2008: 291). Pour le moment, je dois admettre que les causes exactes de l'alternance entre le futur synthétique et le futur périphrastique dans les phrases positives de notre corpus m'échappent. Selon Sankoff et Wagner (2006 : 213), l'emploi du futur synthétique pourrait être dû à un degré très élevé de formalité des entrevues. Je n'ai pas tenu compte de ces divers degrés de formalité et je ne peux donc pas vérifier si tel est le cas dans mon corpus. L'étude de Blondeau (2006) tend à démontrer que l'alternance entre les deux formes du futur s'expliquerait par le biais d'un facteur sémantique (degré de contingence par rapport à l'événement). Je n'ai pas non plus tenu compte de ce facteur lors de l'analyse des données, analyse effectuée avant la publication de l'article de Blondeau (2006) ${ }^{13}$. Je ne peux donc vérifier l'importance de ce facteur intralinguistique, mais il va de soi qu'une telle analyse serait ultérieurement très pertinente.

Enfin, concernant les résultats de la variable «Le présentatif en c'est des », j’ai noté que les hommes hésitaient davantage entre la forme standard et la forme non standard (52,3\% pour la forme neutralisée), alors que les femmes employaient très largement la forme vernaculaire (83,8 \% des cas). Cet écart peut être pour le moins surprenant lorsque l'on considère, à l'instar de Labov (2001 : 266) que : «for stable sociolinguistic variables, women show a lower rate of stigmatized variants and a higher rate of prestige variants than men. » Les résultats obtenus pourraient indiquer que les variantes c'est des et ce sont des ne sont pas stables, ce qui est très probable puisque les résultats généraux montrent qu'il y a encore hésitation entre les deux formes. Labov (2001 : 293) souligne également que : «Women conform more closely than men to sociolinguistic norms that are overtly prescribed, but conform less than men when they are not». Les résultats indiqueraient donc que la

${ }^{13}$ Les précédentes études portant sur l'alternance entre le futur synthétique et le futur périphrastique en français québécois (Deshaies et Laforge1981 ; Émirkanian et Sankoff 1985 ; Zimmer 1994 ; Poplack et Turpin 1999 ; Sankoff et Wagner 2006) n'ayant pas identifié ce facteur comme étant significatif, j’avais donc choisi d'en faire abstraction. 
variante vernaculaire c'est des devient progressivement la forme standard, mais qu'elle n'est pas encore ouvertement prescrite ${ }^{14}$.

\section{Analyse externe des résultats généraux}

Le tableau 3 présente le pourcentage des formes standard utilisées selon le sexe des locuteurs, ainsi que l'indice p (indice de significativité) de la variable sexe.

Tableau 3 : Pourcentages de variantes standard en fonction du sexe des interviewés

\begin{tabular}{||l|c|c|c|}
\hline \hline Sexe & Taux moyens en $\%$ & n/N & p \\
\hline \hline Femmes & 86,2 & $560 / 766$ & \multirow{2}{*}{0,004} \\
\hline Hommes & 77,8 & $2158 / 2775$ & \\
\hline
\end{tabular}

Globalement, les femmes utilisent plus de variantes de référence que les hommes. Elles obtiennent un score de $86,2 \%$ contre $77,8 \%$ pour les hommes. Le seuil significatif du test de Fisher $(0,05)$ étant largement dépassé $(\mathrm{p}=0,004)$, la variable sexe est donc hautement significative. Ce n'est pas un hasard puisque l'on vient de voir que selon Labov (2001: 293), les femmes ont davantage tendance à se conformer à la norme que les hommes pour ce qui est des variables stables.

Tableau 4 : Pourcentages de variantes standard en fonction de l'âge des interviewés

\begin{tabular}{|l|c|c|c|}
\hline Groupes d'âge & Taux moyens en $\%$ & $\mathbf{n} / \mathbf{N}$ & $\mathbf{p}$ \\
\hline \hline 61 ans et plus & 80,1 & $1083 / 1352$ & \multirow{2}{*}{0,0002} \\
\hline 31 ans à 60 ans & 74,8 & $1618 / 2162$ & \\
\hline 30 ans et moins & 62,9 & $17 / 37$ & \\
\hline
\end{tabular}

Le tableau 4 indique que le groupe des «30 ans et moins» est celui qui utilise dans l'ensemble le moins de variantes de référence (62,9\%). Cette catégorie est suivie des «31 à 60 ans » à 74,8\%, puis des « 61 ans et plus » à 80,1\%. La valeur de p est égale à 0,0002, hautement significative. Il existe donc, semble-t-il, une différence significative entre les trois groupes d'âge, différence probablement due aux «30 ans et moins» qui obtiennent le plus faible score. Un nouveau test exact de Fisher

14 Il faut noter que, dans la quatorzième édition du Bon usage de Grevisse-Goosse, l'emploi du futur périphrastique dans les phrases positives ainsi que l'emploi du présentatif en "c'est des » ne sont pas proscrits (Grevisse-Goose 2008: paragraphe 933, pages 1159 et 1042), ce qui indique non seulement que ces usages ne sont pas exclusivement québécois, mais aussi qu'ils ne sont pas forcément considérés comme non standard. 
indique qu'en excluant la catégorie des «30 ans et moins », p est une fois de plus inférieure à 0,05 (p $=0,001)$, ce qui signifie que les deux groupes de locuteurs « 31 ans et plus » et « 61 ans et plus » se distinguent également significativement. Lorsqu'on regarde les résultats du tableau 4, on remarque que plus l'âge des interviewés augmente, plus le pourcentage de variantes de référence s'élève. Le test de Pearson que nous avons effectué (dont le résultat est $\mathrm{p}=0,984$ ) vient confirmer nettement cette corrélation. Thibault (1997:25) précisait, par ailleurs, que :

Si l'on veut suivre l'évolution des normes au sein d'une communauté, normes vernaculaires aussi bien que normes standards, il est donc indiqué de comparer le comportement de cette cohorte [30 à 55 ans] à celui des 60-75, dont le parler est censé représenter un état de langue antérieur, celui de leur jeunesse.

D’après Thibault (1997: 25), la norme grammaticale du français québécois tendrait donc petit à petit vers une utilisation plus prononcée de variantes populaires que de variantes de référence. Le score du groupe des «30 ans et moins» semblerait presque confirmer la présence d'un changement en cours. Toutefois, ce groupe n'est représenté dans notre corpus que par deux locuteurs. Il me paraît donc risqué de conclure sur une telle éventualité. Enfin, il est tout à fait possible que, pour des raisons qui m’échappent encore, les Québécois soient plus à l'aise dans leur utilisation du français québécois standard (ou même qu'ils choisissent délibérément de s’y conformer) au fur et à mesure qu'ils vieillissent.

Tableau 5 : Variantes standard en fonction de la catégorie d'emploi des interviewés

\begin{tabular}{|c|c|c|c|}
\hline Catégorie d'emploi & Taux moyens en $\%$ & $\mathbf{n} / \mathbf{N}$ & $\mathrm{p}$ \\
\hline Éminence religieuse & 84,3 & $70 / 83$ & \multirow{9}{*}{0,225} \\
\hline Juges/Avocats & 79,5 & $213 / 268$ & \\
\hline Universitaires & 78,7 & $422 / 536$ & \\
\hline Hauts-gradés & 78,2 & $90 / 115$ & \\
\hline Responsables & 76,9 & $492 / 640$ & \\
\hline Artistes & 76,4 & $427 / 559$ & \\
\hline Politiciens & 75,9 & $710 / 935$ & \\
\hline Médecins & 74 & $230 / 311$ & \\
\hline Élite sportive & 68 & $64 / 94$ & \\
\hline
\end{tabular}

Concernant la variable catégorie d'emploi, deux groupes semblent se démarquer des autres. Le score des « éminences religieuses » est le plus élevé (84,3\%), celui des « élites sportives » est le plus bas (68 \%). 
Les sept autres catégories d'emploi obtiennent des scores situés entre $74 \%$ et 79,5\%. Cependant, la valeur de p indiquée dans le tableau ci-dessus est égale à 0,225 , ce qui signifie que les locuteurs des neuf catégories ne se distinguent pas significativement lorsque l'on prend l'ensemble des pourcentages des variantes de référence ${ }^{15}$. En d'autres termes, quel que soit leur emploi, les locuteurs semblent utiliser un même modèle normatif de grammaire à l'oral.

\section{Conclusion}

Dans un premier temps, nous avons vu que le débat sur la norme du français au Québec a vite opposé deux principales positions : d'un côté les partisans en faveur d'une norme internationale, de l'autre, les partisans en faveur d'une norme qui tient compte des usages réels au Québec. Nous avons également vu que selon Barbaud (1998a, 1998b), le problème venait de la concurrence des formes grammaticales standard et vernaculaires dans le discours des élites québécoises. Il affirmait d'ailleurs que si la norme linguistique du français québécois oral devait être basée sur le parler de l'élite, cela poserait d'énormes problèmes communicationnels avec le reste de la francophonie et qu'il y aurait une diglossie entre l'oral et l'écrit au Québec. Mon étude démontre que cela ne peut pas être le cas, puisque les membres des élites sociale et culturelle du Québec emploient de façon homogène un modèle grammatical oral très proche de celui présenté dans Le bon usage (donc de l'écrit).

Dans une étude parue récemment, Maurais souligne que les Québécois «croient que leur variété de langue tend à se rapprocher des autres variétés de français » (Maurais 2008: 113). Les résultats obtenus à partir de l'examen du corpus Le Point tendent à démontrer que c'est le cas pour ce qui est de leur norme grammaticale orale. Ces mêmes résultats viennent aussi appuyer directement les positions de Corbeil (1993, 2007), de Nemni (1998) et du CLF (2007).

J'ai néanmoins quelques réserves concernant certains des résultats obtenus à partir de mon corpus. Si les tests statistiques que j'ai effectués permettent de tenir compte des données malgré le nombre peu élevé de locuteurs, il faut rester prudent face aux chiffres concernant les catégories « élites sportives », « éminences religieuses » et « hauts gradés » qui sont représentées au maximum par trois personnes, ainsi que ceux du groupe d'âge «30 ans et moins » (deux personnes).

\footnotetext{
15 Je tiens également à souligner que deux autres groupes tendent à se distinguer (de par leur emploi plus important de formes de type vernaculaire) malgré des différences statistiquement non significatives : les élites sportives (pour les variables: tout/tous, les relatives et interrogatives indirectes et le futur) et les hauts gradés (pour fait, le présentatif, les relatives et interrogatives indirectes et l'insertion du $P$ non étymologique). Il serait intéressant de voir si cela est dû ou non au facteur niveau de scolarité que je n'ai pu prendre en compte.
} 
J'ai certes pris en compte quatorze variables grammaticales, mais plusieurs autres tournures mériteraient notre attention (les prépositions orphelines, la féminisation des mots à initiale vocalique, etc.). Par ailleurs, une analyse de type Goldvarb (Tagliamonte 2006) ou régression multiple permettrait de rendre compte de l'importance de chaque facteur externe dans la variation des phénomènes observés: Le facteur âge est-il plus pertinent que le facteur sexe ? Si oui, dans quelle proportion? Ces limites constituent en soi des faiblesses, sans pour autant me faire manquer mon objectif principal qui était de déterminer s'il existe oui ou non un modèle de grammaire orale commun aux membres des élites québécoises. Je suis convaincu que c'est effectivement le cas.

Aujourd'hui, si l'AQPF reposait la question «Quel français doit-on enseigner au Québec ?», tout porte à croire que ce sont les formes prescrites dans des grammaires telles que Le bon usage de Grevisse-Goosse qui doivent être enseignées, puisque celles-ci font partie des tournures normatives employées par les élites québécoises dans un registre soutenu. L’un des problèmes majeurs de l'enseignement du français au Québec n'est d'ailleurs pas, je crois, l'enseignement des formes standard, mais plutôt l'enseignement et la maitrise des registres qui composent notre variété, tant au niveau du lexique, que de la prononciation et de la grammaire. Il ne s'agit certainement pas d'enrayer les différences dialectales qui séparent le français québécois du français de France (ou du français international), mais plutôt d'insister sur ce qui nous rassemble et ce qui nous éloigne selon les contextes de communication.

Enfin, si Lamonde (2004) fustige le parler des élites québécoises, mon étude de corpus montre qu'il n'y a pas de raison de le faire. Je terminerai d'ailleurs en allant totalement à l'encontre de sa position en proposant que la norme du français québécois soit le modèle socialement valorisé que les élites québécoises (et non plus la majorité des Québécois comme le suggère la définition de l'AQPF 1977) tendent à utiliser dans les situations de communication formelle. Pour l'instant, je suis convaincu que, pour ce qui est de la grammaire orale comme écrite, ce modèle est bien celui du français de référence décrit entre autres dans Le bon usage de Grevisse-Goosse.

\section{Références bibliographiques}

Association québécoise des professeurs de français. 1977. Le congrès du dixième anniversaire : Les résolutions de l'assemblée générale. Québec français $28: 10-12$.

Barbarie, Yves. 1982. Analyse sociolinguistique de la syntaxe de l'interrogation en français québécois. Revue québécoise de linguistique 12(1) : 145-168.

Barbaud, Philippe. 1987a. Le français sans façon. Montréal : Hurtubise. 
Barbaud, Philippe. 1987b. Parlerons-nous cajun? Dans L'avenir du français au Québec. Montréal: Québec-Amérique, 61-70

Barbaud, Philippe. 1998a. Dissidence du français québécois et évolution dialectale. Revue québécoise de linguistique 26 (2) : 107-128.

Barbaud, Philippe. 1998b. Tendances lourdes du français québécois. Dans Français d'Amérique. Variation, créolisation, normalisation, sous la direction de Patrice Brasseur, 17-36. Avignon: CECAV.

Bélanger, Gaëlle. 2003. Propriétés adverbiales du quantifieur TUT en français québécois : critique syntaxique et sémantique. Mémoire de maitrise, Université du Québec.

Bigot, Davy. 2008. "Le point» sur la norme grammaticale du français québécois oral. Thèse de doctorat, Université du Québec.

Blondeau, Hélène 2006. La trajectoire de l'emploi du futur chez une cohorte de Montréalais francophones entre 1971 et 1995. Dans Les variétés de français en Amérique du Nord. Évolution, innovation et description, sous la direction de Robert A. Papen et Gisèle Chevalier. Numéro conjoint de la Revue canadienne de linguistique appliquée 9(2), Revue de l'Université de Moncton 37(2): 73-98.

Bouchard, Pierre et Jacques Maurais. 2001. Norme et médias. Les opinions de la population québécoise. Terminogramme. Norme et médias 97-98 : 111-126.

Boulanger, Jean-Claude et Alain Rey, dir. 1992. Dictionnaire québécois d'aujourd'bui : langue française, histoire, géographie, culture générale. Saint-Laurent : Dicorobert.

Cajolet-Laganière, Hélène et Pierre Martel. 1995. La qualité de la langue au Québec. Dans la collection Diagnostic 18. Québec : IQRC.

Chaudenson, Robert. 1997. Acrolecte. Dans Sociolinguistique. Concepts de base, sous la direction de Marie-Louise Moreau, 100-101. Liège : Mardaga.

Comité interministériel sur la situation de la langue française. 1996. Le français langue commune. Enjeu de la société québécoise. Québec: Direction des communications du ministère de la Culture et des Communications.

Commission des États généraux sur la situation et l'avenir de la langue française au Québec. 2001. Le français, une langue pour tout le monde. Québec: Gouvernement du Québec.

Conseil supérieur de la langue française. 2007. Bulletin, vol. 23, nº 1, juin 2007.

Conseil de la langue française. 1990. L'aménagement de la langue : pour une description du français québécois. Québec : Conseil de la langue française.

Corbeil, Jean-Claude. 1993. Le français au Québec, une langue à restaurer? Vie pédagogique $86: 27$ 30.

Corbeil, Jean-Claude. 2007. L'embarras des langues. Origine, conception et évolution de la politique linguistique québécoise. Montréal : Québec Amérique.

Cox, Terry B. 1998. Vers une norme pour un cours de phonétique française au Canada. Revue canadienne des langues vivantes 54(2) : 175-197.

Cyr, Francine. 1991. La quantification à distance en français québécois. Mémoire de maîtrise, Université de Montréal. 
Daveluy, Michelle. 1987. L'usage des déterminants démonstratifs dans la communauté francophone de Montréal en 1971 et 1984 . Mémoire de maîtrise, Université de Montréal.

Daveluy, Michelle. 2005. Les langues étendards. Allégeances langagières en français parlé à Montréal. Dans la collection Langue et pratiques discursives. Québec : Nota bene.

De Cat, Cécile. 2000. Towards a unified analysis of French floating quantifiers. Journal of French language studies $10: 1-25$.

Deshaies, Denise et Eric Laforge. 1981. Le futur simple et le futur proche dans le français parlé dans la ville de Québec. Langues et linguistique 7 : 21-37.

Emirkanian, Louisette et David Sankoff. 1985. Le futur simple et le futur périphrastique. Dans Les tendances dynamiques du français parlé à Montréal T.1, sous la direction de Monique Lemieux et Henrietta Cedergren, 189-204. Québec: Gouvernement du Québec, Office de la langue française.

Forget, Danielle. 2008. Quel est le français standard au Québec. Dans Le français parlé: études sociolinguistiques, sous la direction de Pierrette Thibault, 153-16. Edmonton : Linguistic Research Inc. [1979]

Grevisse, Maurice et André Goosse. 2008. Le bon usage. Grammaire française avec des remarques sur la langue française d'aujourd'bui. $14^{\mathrm{e}}$ édition. Gembloux : Duculot.

Junker, Marie-Odile. 1994. French universal quantifiers in conceptual semantics. Linguistics 32(2) : 213-239.

Junker, Marie-Odile. 1995. Syntaxe et sémantique des quantificateurs flottants tous et chacun: distributivité en sémantique conceptuelle. Genève : Librarie Droz.

Kemp, William. 1979. L'histoire récente de ce que, qu'est-ce que et qu'osque à Montréal: trois variantes en interaction. Dans Le français parlé: études sociolinguistiques, sous la direction de Pierrette Thibault, 53-74. Edmonton : Linguistic Research Inc.

Labov, William. 2001. Principles of linguistic change, Volume 2 : Social factors. Oxford : Blackwell.

Lamonde, Diane. 2004. Anatomie d'un joual de parade. Le bon français d'ici par l'exemple. Montréal : Varia.

Lavoie, Réal. 1989. Morphologie des variantes du futur et du conditionnel dans le parler québécois. Revue québécoise de linguistique théorique et appliquée 8(3-4), 189-205.

Léard, Jean-Marcel. 1995. Grammaire québécoise d'aujourd'bui. Comprendre les québécismes. Montréal: Guérin.

Léard, Jean-Marcel et Normand Beauchemin. 1991. Quelques propriétés morpho-syntaxiques du français du Québec : l'interprétation de /tUt/. Dans Actes du $3^{e}$ colloque international Français de France-Français du Canada, sous la direction de Hans-Joseph Niederehe et Lothar Wolf, 179-199. Tübingen : Niemeyer.

Lemieux, Monique. 1982. M'as / tut/ vous conter ça. Dans La syntaxe comparée du français standard et populaire: approches formelle et fonctionnelle, sous la direction de Claire Lefebvre, 49-71. Québec: OLF.

Lemieux-Nieger, Monique, Leblanc Louise et Paquin Sylvie. 1981. La variation dans l'emploi de /tu/ et de /tUt/ dans le français parlé à Montréal. Dans Variation Omnibus, sous la direction de David Sankoff et Henrietta Cedergren, 313-319. Edmonton : Linguistic Research Inc. 
Lemieux, Monique et David Sankoff. 1983. On peux-tu /tUt/ désambiguiser? Revue de l'association québécoise de linguistique 3(2), 159-164.

Lemieux, Monique, St-Amour Marielle et David Sankoff. 1985. /TUT/ en français de Montréal : un cas de neutralisation morphologique. Dans Les tendances dynamiques du français parlé à Montréal, T.2, sous la direction de Monique Lemieux et Henrietta Cedergren, 7-89. Québec: Gouvernement du Québec.

Lepicq, Dominique et Richard Bourhis. 1995. Aménagement linguistique et norme langagière au Québec. Linx 2(33) : 109-128.

Maleville, Mireille. 1982. Du barbarisme à la sociabilité ou ce qui joue un rôle dans l'établissement des normes. Le français dans le monde $169:$ 67-72.

Maurais, Jacques. 1999. La qualité de la langue, un projet de société. Québec: Conseil de la langue française.

Maurais, Jacques. 2005. La langue des bulletins d’information à la radio québécoise : premier essai d'évaluation. Dans la collection Suivi de la situation linguistique, Étude 6. Québec: Gouvernement du Québec.

Maurais, Jacques. 2008. Les Québécois et la norme. L'évaluation par les Québécois de leurs usages linguistique. Dans la collection Suivi de la situation linguistique, Étude 7. Québec: Gouvernement du Québec.

Meney, Lionel. 1999. Dictionnaire québécois français. Mieux se comprendre entre francophones. Montréal: Guérin.

Meney, Lionel. 2003. Réaffirmation que la langue du Québec est bien le français. Le Soleil 8, A11.

Meney, Lionel. 2005a. Un autre dictionnaire québécois, pourquoi? Le Devoir 7. http://www.ledevoir.com/2005/01/07/72023.html, 2005a.

Meney, Lionel. 2005b. L'inquiétante hostilité québécoise au français. Le monde 21 mars :13.

Meney, Lionel. 2010. Main basse sur la langue. Idéologie et interventionnisme linguistique au Québec. Montréal : Liber.

Ministère des affaires culturelles du Québec. 1965. Norme du français parlé et écrit an Québec. Québec: Office de la langue française, Gouvernement du Québec.

Morin, Yves-Charles. 1982. De quelques [1] non étymologiques dans le français du Québec. Revue québécoise de linguistique 11(2), 9-48.

Mougeon, Raymond. 1994. Recherche sur les origines de la variation vas, m'as, vas en français québécois. Dans Français du Canada, français de France, sous la direction de Thomas Lavoie, 6077. Tübingen : Niemeyer.

Nemni, Monique. 1998. Le français au Québec : représentation et conséquences pédagogiques. Revue québécoise de linguistique 26(2) : 151-175.

Ostiguy, Luc, Éric Champagne, Flore Gervais et Monique Lebrun. 2005. Le français oral soutenu chez des étudiants québécois en formation pour l'enseignement au secondaire. Dans la collection Suivi de la situation linguistique, Étude 4. Québec : Gouvernement du Québec.

Poplack, Shana et Danielle Turpin. 1999. Does the Futur have a future in (Canadian) French. Probus 11 : 133-164. 
Reinke, Kristin. 2005. La langue à la télévision québécoise : aspect sociophonétiques. Dans la collection Suivi de la situation linguistique, Étude 6. Québec : Gouvernement du Québec, 2005.

Remysen, Wim. 2003. Le français au Québec: au-delà des mythes. Romaneske 1. http://www.kuleuven.ac.be/vlr/031quebec.htm.

Sankoff, Gillian et Pierrette Thibault. 1977. L'alternance entre les auxiliaires avoir et être dans le français parlé à Montréal. Langue française 34 : 81-108.

Sankoff, Gillian et Suzanne E. Wagner. 2006. Age grading in retrograde movement : the inflected future in Montreal French. University of Pennsylvania working papers in Linguistics 12(2) : 203-216.

Tagliamonte, Sali. 2006. Analysing sociolinguistics variation. Cambridge : Cambridge University Press.

Thibault, Pierrette. 1997. Âge. Dans Sociolinguistique. Concepts de base, sous la direction de Marie-Louise Moreau, 20-24. Liège : Mardaga.

Villers, Marie-Éva de. 2003. Multi dictionnaire de la langue française. $4^{\mathrm{e}}$ édition. Montréal: Québec Amérique.

Zimmer, David. 1994. Ça va tu marcher, ça marchera tu pas, je le sais pas : le futur simple et le futur périphrastique dans le français parlé à Montréal. Langues et linguistique 20 : 213-226. 